University of Nebraska - Lincoln

DigitalCommons@University of Nebraska - Lincoln

$4-21-2021$

\title{
A Guide to Helminth Parasites Reported from Shorebirds (Charadriidae) from the Americas
}

\author{
Albert G. Canaris \\ Hamilton, Montana, agcanaris@gmail.com \\ Sofia Capasso \\ Centro de Estudios Parasitológicos y de Vectores (CEPAVE), Bueno Aires, Argentina, \\ capasso.sofia@gmail.com
}

Gay J. Canaris

Billings Clinic, Billings, Montana, htcranch@gmail.com

Follow this and additional works at: https://digitalcommons.unl.edu/manter

Part of the Biodiversity Commons, Parasitology Commons, and the Zoology Commons

Canaris, Albert G.; Capasso, Sofia; and Canaris, Gay J., "A Guide to Helminth Parasites Reported from Shorebirds (Charadriidae) from the Americas" (2021). MANTER: Journal of Parasite Biodiversity. 16. https://digitalcommons.unl.edu/manter/16

This Article is brought to you for free and open access by the Parasitology, Harold W. Manter Laboratory of at DigitalCommons@University of Nebraska - Lincoln. It has been accepted for inclusion in MANTER: Journal of Parasite Biodiversity by an authorized administrator of DigitalCommons@University of Nebraska - Lincoln. 


\title{
A Guide to Helminth Parasites Reported from Shorebirds (Charadriidae) from the Americas
}

\author{
Albert G. Canaris, ${ }^{1}$ Sofia Capasso, ${ }^{2}$ and Gay J. Canaris ${ }^{3}$ \\ 1 Hamilton, Montana, USA \\ 2 Centro de Estudios Parasitológicos y de Vectores (CEPAVE), CCT La Plata (CONICET-UNLP), La Plata, Buenos Aires, Argentina \\ 3 Billings Clinic, Billings, Montana, USA
}

\section{Contents}

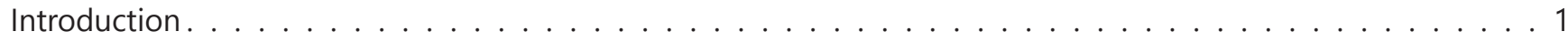

Table I. Checklist of helminth parasites reported for the shorebird family Charadriidae from the Americas . . . . 2

Table II. Trematode parasite-host list for shorebird family Charadriidae of the Americas . . . . . . . . . . 8

Table III. Cestode parasite-host list for shorebird family Charadriidae from the Americas . . . . . . . . . . . . 10

Table IV. Nematode parasite-host list for shorebird family Charadriidae of the Americas . . . . . . . . . . . 11

Table V. Acanthocephala parasite-host list for shorebird family Charadriidae of the Americas . . . . . . . 12

Tables VI. (A, B, C, and D) Summary data compiled from Tables I-V and Literature Cited . . . . . . . . . . . . 13

Literature Cited . . . . . . . . . . . . . . . . . . . . . . . . . . . . . . . . 15

\section{Introduction}

The shorebird family Charadriidae in the Americas consists of 21 native and 7 vagrant species. Members of the family occupy a diversity of open habitats, ranging from Arctic tundra during nesting, coastal sands, and mudflats to inland prairies, savannas, and wetlands. Some native plovers migrate from nesting grounds in North America to wintering grounds in South America (Hayman et al., 1986; Paulson, 2005; Winkler et al., 2020).

Our search of the literature revealed the following: 17 of 28 host species infected with helminth parasites, 153 helminth species, and 199 infections involving 13 geographic areas. The purpose of this guide is to provide easy access to this data and information relevant to helminth infections in charadriids from the Americas. Information is summarized in Tables I-VI.
Table I lists in sequence host, parasite, geographic location, and attenuated citation. Common names are given for each host. Host names are listed alphabetically, and older scientific names used in the literature search are in parentheses. Host geographic distribution is abbreviated as follows: NA = North America, $\mathrm{M}$ $=$ Mexico, $C A=$ Central America, $S A=$ South America, $A=$ Americas $(N A+M+C A+S A)$. If present, parasite species are listed in the following order: trematode, cestode, nematode, and acanthocephala. The helminth species names are listed as they were given in the cited literature.

Tables II-V are parasite-host lists for trematode, cestode, nematode, and acanthocephalan species and host of the species associated with the parasite. Table $\mathrm{VI}$ is a summary of information extracted from the tables and literature cited section. 
Table I. Checklist of helminth parasites reported for the shorebird family Charadriidae from the Americas

Native hosts in the Americas Parasite Geographic location Citation

\section{Snowy Plover A}

Charadrius nivosus (alexandrinus) Paramaritremopsis solielangi Gyrocoelia miligani

Shipleyia inermis

Nadejdolepis litoralis

Nadejdolepis paranitidulans

Puna Plover SA

Charadrius alticola

Collared Plover A

Charadrius collaris

Two-Banded Plover SA

Charadrius falklandicus

Ringed Plover NA

Charadrius hiaticula

Piping Plover NA

Charadrius melodus

Charadrius modestus (Zonibyx)

Mountain Plover NA, M

Charadrius montanus

(Podasocys and Eupoda)

Semipalmated Plover A

Charadrius semipalmatus
No reference found

Gyrocoelia crassa

Gyrocoelia fuhrmani

Hymenolepis collariella

Hymenolepis hughesi

Taenia charadrii

Sciadiocara umbellifera

Stellocaronema skrjabini

Notocotylus chionis

Odhneria odhneri

Tetrameres megaphasmidiata

Paramonostomum actidis

Aploparaksis brachyphallos

Eulimdana lari

Eulimdana sonni

Gynaecotyla sippiwissettensis

Hymenolepis hughesi

Progynotaenia americana

Chevreuxia americana

Porrocaecum falklandicus

Florida

California, Texas, Mexico

Cyclocoelum obscurum

Echinochasmus sp.

Notocotylus stagnicolae

Levinseniella carcinidis

Levinseniella cruzi

Maritrema gratiosum

Maritrema ovatum

Microphallus claviformes

Microphallus kinsellai

Paramaritremopsis solielangi

Paramonostomum actidis

Andes

Mexico, Texas

Guadeloupe

Brazil

Peru

Peru

Argentina-Patagonia

Argentina-Patagonia

Argentina-Patagonia

Puerto Rico

North America

North America?

USA

Texas; Guadeloupe

Cuba
Florida
USA
USA
California
Oklahoma, USA
North America
Puerto Rico
Belize
Belize
Puerto Rico

Great Plains, Arizona,

Belize

Michigan, Texas

Michigan, Texas

Belize

Belize

Hayman, P., et al. 1986

Central and South America Yamaguti, S. 1959

Mexico, South America Rêgo, A. A. 1968

Coil, W. H. 1956; Deblock, S., and F. Rosé. 1962

Graber, M., and J. Euzeby. 1976b

Hartwich, G., and I. Kilias. 1992

Wong, P. L., and R. C. Anderson. 1991

Wong, P. L., and R. C. Anderson. 1991

Capasso, S., et al. 2017

Capasso, S., et al. 2017

Cremonte, F. M., et al. 2001

Thatcher, V. E. 1993

Yamaguti, S. 1959

Hoberg, E. P. 1986

Bartlett, C. M. 1992

Yamaguti, S. 1971

Webster, J. D. 1947;

Graber, M., and J. Euzeby. 1976a;

Graber, M., and J. Euzeby. 1976b

Webster, J. D. 1947

Wong, P. L., and R. C. Anderson. 1991

Yamaguti, S. 1961

Paulson, D. 2005
Macko, J. K. 1969

Loftin, H. 1960

Yamaguti, S. 1971

Doss, M. A., and M. M. Farr. 1969a

Yamaguti, S. 1971

Yamaguti, S. 1971

Yamaguti, S. 1971

Cable, R. M., et al. 1960

Canaris, A. G., and J. M. Kinsella. 2001

Canaris, A. G., and J. M. Kinsella. 2001

Cable, R. M., et al. 1960 


\section{Table I. (cont.) Helminth parasites reported for the shorebird family Charadriidae from the Americas}

\begin{tabular}{|c|c|c|c|}
\hline & Hymenolepis hughesi & Guadeloupe & Graber, M., and J. Euzeby. 1976b \\
\hline & Nadejdolepis litoralis & Belize & Canaris, A. G., and J. M. Kinsella. 2001 \\
\hline & Paraliga charadrii & $\begin{array}{l}\text { Manitoba, } \\
\text { New Brunswick, Canada, } \\
\text { Delaware, USA, Venezuela }\end{array}$ & Didyk, A. S., and M. D. B. Burt. 1998 \\
\hline & Progynotaenia americana & Texas; Belize & $\begin{array}{l}\text { Webster, J. D. 1951; } \\
\text { Canaris, A. G., and J. M. Kinsella. } 2001\end{array}$ \\
\hline & Chevreuxia americana & Florida, USA & Wong, P. L., and R. C. Anderson. 1991 \\
\hline & Skrjabinoclava horrida & Cuba & Barus, V., and N. L. Hernandez. 1971 \\
\hline & Skrjabinoclava morrisoni & Canada, USA & Wong, P. L., and R. C. Anderson. 1990b \\
\hline & Skrjabinoclava pusillae & $\begin{array}{l}\text { Alberta, Saskatchewan, } \\
\text { Manitoba, Ontario, New } \\
\text { Brunswick, Nova Scotia; } \\
\text { Florida, Texas }\end{array}$ & $\begin{array}{l}\text { Wong, P. L., et al. 1990; } \\
\text { Wong, P. L., and R.C. Anderson. 1990b }\end{array}$ \\
\hline & Skrjabinoclava semipalmatae & Texas; California & $\begin{array}{l}\text { Wong, P. L., and R. C. Anderson. 1987; } \\
\text { Wong, P. L., and R. C. Anderson. 1990b }\end{array}$ \\
\hline & Skrjabinoclava sp. & $\begin{array}{l}\text { Alberta, Saskatchewan, } \\
\text { Manitoba, Ontario, } \\
\text { New Brunswick, } \\
\text { Nova Scotia, California }\end{array}$ & Wong, P. L., et al. 1990 \\
\hline & Skrjabinoclava tupacincai & New Brunswick & Wong, P. L., and R. C. Anderson. 1990b \\
\hline & Stellocaronema skrjabini & $\begin{array}{l}\text { Puerto Rico; Alberta, } \\
\text { Manitoba, California, } \\
\text { Texas, USA, Peru }\end{array}$ & $\begin{array}{l}\text { Whittaker, F. H., et al. 1970; } \\
\text { Wong, P. L., and R. C. Anderson. } 1991\end{array}$ \\
\hline & Viktorocara capillaris & $\begin{array}{l}\text { Alberta, Manitoba; } \\
\text { Ontario }\end{array}$ & $\begin{array}{l}\text { Wong, P. L., et al. 1990; } \\
\text { Wong, P. L., and R. C. Anderson. } 1991\end{array}$ \\
\hline & Viktorocara limosae & $\begin{array}{l}\text { Alberta, Manitoba; } \\
\text { California, USA }\end{array}$ & $\begin{array}{l}\text { Wong, P. L., et al. 1990; } \\
\text { Wong, P. L., and R. C. Anderson. } 1991\end{array}$ \\
\hline & Plagiorhynchus charadrii & Belize & Canaris, A. G., and J. M. Kinsella. 2001 \\
\hline \multicolumn{4}{|l|}{ Killdeer A } \\
\hline \multirow[t]{21}{*}{ Charadrius vociferous (Oxyechus) } & Collyriclum sp. & Oklahoma & Riggins, M. A. 1953 \\
\hline & Cyclocoelum lanceolatum & $\begin{array}{l}\text { California; Colorado; } \\
\text { New Mexico, Texas }\end{array}$ & $\begin{array}{l}\text { Hannum, C. A., and B. E. Wilson. 1934; } \\
\text { Eckman, M. K. 1968; Blaylock, R. B. } 1989\end{array}$ \\
\hline & Cyclocoelum obscurum & Colorado & Blaylock, R. B. 1989 \\
\hline & Cyclocoelum triangularis & Oklahoma & Riggins, M. A. 1953 \\
\hline & Echinoparyphium aconiatum & $\begin{array}{l}\text { Colorado, New Mexico, } \\
\text { Texas }\end{array}$ & Blaylock, R. B. 1989 \\
\hline & Echinoparyphium baculus & Texas & Blaylock, R. B. 1989 \\
\hline & Echinostoma sp. & Colorado & Eckman, M. K. 1968 \\
\hline & Microphallus quechuasensis & Peru & Caballero, C. E., and H. N. Ibáñez. 1970 \\
\hline & Notocotylus attenuatus & Colorado, New Mexico & Blaylock, R. B. 1989 \\
\hline & Plagiorchis vitellatus & Colorado & Blaylock, R. B. 1989 \\
\hline & Stomylotrema vicarium & Florida & Forrester, D. J., and M. G. Spalding. 2003 \\
\hline & Tanaisia fedtschenkoi & Georgia; Colorado; & Byrd, E. E., and J. F. Denton. 1950; \\
\hline & & British Columbia; & Eckman, M. K. 1968; Jensen, D. N. 1971; \\
\hline & & New Mexico, Texas; & Blaylock, R. B. 1989; \\
\hline & & Florida & Forrester, D. J., and M. G. Spalding. 2003 \\
\hline & Transcoelom sp. & Colorado & Blaylock, R. B. 1989 \\
\hline & Uvitellina macroisophaga & California; Oklahoma & $\begin{array}{l}\text { Hannum, C. A., and B. E. Wilson. 1934; } \\
\text { Riggins, M. A. } 1953\end{array}$ \\
\hline & Uvitellina psuedocotylea & USA & Doss, M. A., and M. M. Farr. 1969b \\
\hline & Anomotaenia bacilligera & Oklahoma & Riggins, M. A. 1953 \\
\hline & Anomotaenia filovata & $\begin{array}{l}\text { Nebraska; Colorado; } \\
\text { New Mexico, Texas }\end{array}$ & $\begin{array}{l}\text { Clark, D. T. 1952; Eckman, M. K. 1968; } \\
\text { Blaylock, R. B. } 1989\end{array}$ \\
\hline & Aploparaksis sp. & Colorado; Texas & Eckman, M. K. 1968; Blaylock, R. B. 1989 \\
\hline
\end{tabular}




\section{Table I. (cont.) Helminth parasites reported for the shorebird family Charadriidae from the Americas}

\begin{tabular}{|c|c|c|c|}
\hline Native hosts in the Americas & Parasite & Geographic location & Citation \\
\hline & Choanotaenia macracantha & Oklahoma & Riggins, M. A. 1953 \\
\hline & Choanotaenia sp. & Colorado & Eckman, M. K. 1968 \\
\hline & Gyrocoelia miligani & Antigua; Oklahoma & Baer, J. G. 1940; Riggins, M. A. 1953 \\
\hline & Megalacanthus sp. & Florida & Forrester, D. J., and M. G. Spalding. 2003 \\
\hline & Monopylidium sp. & $\begin{array}{l}\text { Colorado, New Mexico, } \\
\text { Texas }\end{array}$ & Blaylock, R. B. 1989 \\
\hline & Progynotaenia americana & Oklahoma; Oregon, & Riggins, M. A. 1953; \\
\hline & & $\begin{array}{l}\text { Washington; Colorado; } \\
\text { Florida: Cuba }\end{array}$ & Weatherly, N. F., and A. G. Canaris. 1961; \\
\hline & & & $\begin{array}{l}\text { Forrester, D. J., and M. G. Spalding. 2003; } \\
\text { Macko, J. K., and M. Spakulova. } 1998\end{array}$ \\
\hline & Proterogynotaenia neoarctica & Michigan, Texas; & Webster, J. D. 1951; \\
\hline & & Oklahoma; & Riggins, M. A. 1953; \\
\hline & & Colorado & Eckman, M. K. 1968 \\
\hline & Proterogynotaenia odhneri & Cuba & Macko, J. K., and M. Spakulova. 1998 \\
\hline & Capillaria contorta & Colorado; Texas & Eckman, M. K. 1968; Blaylock, R. B. 1989 \\
\hline & Chevreuxia americana & Colorado, New Mexico, & Blaylock, R. B. 1989; \\
\hline & & Texas; Alberta, Manitoba & Wong, P. L., et al. 1990 \\
\hline & Chevreuxia sp. & Colorado & Eckman, M. K. 1968 \\
\hline & Chevreuxia vanelli & Florida & Forrester, D. J., and M. G. Spalding. 2003 \\
\hline & Dispharynx nasuta & Canada; Florida & Wong, P. L., et al. 1990; \\
\hline & & & Forrester, D. J., and M. G. Spalding. 2003 \\
\hline & Dispharynx spiralis & Colorado & Eckman, M. K. 1968 \\
\hline & Oxyspirura sp. & Texas & Blaylock, R. B. 1989 \\
\hline & Schistorophus laciniatus & Oklahoma & Riggins, M. A. 1953 \\
\hline & Skrjabinocerca prima & Alberta & Wong, P. L., et al. 1990 \\
\hline & Spirurid larvae & Florida & Forrester, D. J., and M. G. Spalding. 2003 \\
\hline & Spiruroidean nematode & Oklahoma & Riggins, M. A. 1953 \\
\hline & Stellocaronema skrjabini & Texas & Wong, P. L., and R. C. Anderson. 1991 \\
\hline & Streptocara sp. & Alberta & Wong, P. L., et al. 1990 \\
\hline & Strongyloides sp. & Colorado, New Mexico, & Blaylock, R. B. 1989; \\
\hline & & Texas; Florida & Forrester, D. J., and M. G. Spalding. 2003 \\
\hline & Viktorocara capillaris & Alberta, Manitoba, Ontari & Wong, P. L., et al. 1990 \\
\hline & Acanthocephalan larvae & Florida & Forrester, D. J., and M. G. Spalding. 2003 \\
\hline & Mediorhynchus papillosus & Colorado & Eckman, M. K. 1968 \\
\hline & Mediorhynchus robustus & Oklahoma & Riggins, M. A. 1953 \\
\hline & Polymorphus minutus & Colorado; Texas & Eckman, M. K. 1968; Blaylock, R. B. 1989 \\
\hline Wilson's Plover A & & & \\
\hline Charadrius wilsonia (Pagolla) & Acanthoparyphium pagollae & Puerto Rico & Cable, R. M., et al. 1960 \\
\hline & Gynaecotyla adunca & USA & Doss, M. A., and M. M. Farr. 1969a \\
\hline & Levinseniella carteretensis & North Carolina & Coil, W. H., and R. Heard. 1966 \\
\hline & Levinseniella hunteri & Louisiana & Heard III, R. W. 1968 \\
\hline & Levinseniella leptophallus & Puerto Rico & Cable, R. M., et al. 1960 \\
\hline & Maritreminoides patulum & Puerto Rico & Cable, R. M., et al. 1960 \\
\hline & Mecynophallus glandulosus & Puerto Rico & Cable, R. M., et al. 1960 \\
\hline & Megalophallus reamsi & Florida Keys & Overstreet, R. M., and R. W. Heard III. 1995 \\
\hline & Microphallus claviformes & Puerto Rico & Cable, R. M., et al. 1960 \\
\hline & Neivaia cymbium & Brazil & Fernandes, B. M. M., et al. 2015 \\
\hline & Odhneria odhneri & Georgia & Sinclair, N. R. 1971 \\
\hline & Paramonostomum actidis & Puerto Rico & Cable, R. M., et al., 1960 \\
\hline & Parorchis holotestis & Puerto Rico & Cable, R. M., et al. 1960 \\
\hline & Pseudospelotrema charadrii & Puerto Rico & Cable, R. M., et al. 1960 \\
\hline & Tanaisia valida & Northern Colombia & Rietschel, G., and B. Werding. 1978 \\
\hline & Zygocotyle lunata & North America; Brazil & Yamaguti, S. 1971; \\
\hline & & & Fernandes, B. M. M., et al. 2015 \\
\hline & Gyrocoelia pagollae & North Carolina, Texas; & Coil, W. H. 1963; \\
\hline
\end{tabular}




\section{Table I. (cont.) Helminth parasites reported for the shorebird family Charadriidae from the Americas}

\begin{tabular}{|c|c|c|c|}
\hline Native hosts in the Americas & Parasite & Geographic location & Citation \\
\hline & & Puerto Rico & Cable, R. M., and R. M. E. Myers. 1956 \\
\hline & Paraprogynotaenia jimenezi & Cuba & Rysavy, B. 1996 \\
\hline & Ancyracanthopsis winegardi & Texas & Wong, P. L., and R. C. Anderson. 1990b \\
\hline & Sciadiocara umbellifera & Cuba; Texas & $\begin{array}{l}\text { Barus, V., and N. L. Hernandez. 1971; } \\
\text { Wong, P. L., and R. C. Anderson. } 1991\end{array}$ \\
\hline & Skrjabinoclava horrida & Texas; Cuba & $\begin{array}{l}\text { Wong, P. L., and R. C. Anderson. 1983; } \\
\text { Barus, V., and N. L. Hernandez. } 1971\end{array}$ \\
\hline & Skrjabinoclava pusillae & New Brunswick, Canada & Wong, P. L., and R. C. Anderson. 1987 \\
\hline & Skrjabinoclava semipalmatae & California & Wong, P. L., and R. C. Anderson. 1987 \\
\hline & Skrjabinoclava wilsonae & Texas & Wong, P. L., and R. C. Anderson. 1987 \\
\hline & Tropisurus nouveli & Cuba & Barus, V., and N. L. Hernandez. 1971 \\
\hline & Viktorocara capillaris & Texas & Wong, P. L., and R. C. Anderson. 1990b \\
\hline & Viktorocara schejkini & Cuba & Barus, V., and N. L. Hernandez. 1971 \\
\hline & Plagiorhynchus crassicolle & Cuba & Barus, V., and N. L. Hernandez. 1971 \\
\hline \multirow[t]{5}{*}{ Charadrius sp. } & Leucochloridium propinqua & North America & Yamaguti, S., 1971 \\
\hline & Spelotrema excellens & USA & Yamaguti, S., 1971 \\
\hline & Tanaisia fedtschenkoi & USA & Yamaguti, S., 1971 \\
\hline & Capillaria contorta & $\begin{array}{l}\text { North America, } \\
\text { South America }\end{array}$ & Yamaguti, S. 1961 \\
\hline & Porrocaecum ensicaudatum & North America & Yamaguti, S. 1961 \\
\hline \multicolumn{4}{|l|}{ Tawny-throated Dotterel SA } \\
\hline Oreopholus ruficollis & Oxynema sp. & Argentina & Drago, F. B., et al. 2020 \\
\hline \multicolumn{4}{|l|}{ Diademed Plover SA } \\
\hline Phegornis mitchellii & No reference found & $\begin{array}{l}\text { Andes in Argentina, } \\
\text { Chile, Peru }\end{array}$ & Hayman, P., et al. 1986 \\
\hline \multicolumn{4}{|l|}{ American Golden Plover A } \\
\hline \multirow[t]{7}{*}{ Pluvialis dominica } & Levinseniella propinqua & British Columbia, Canada & Ching, H. L. 1965 \\
\hline & Progynotaenia americana & Canada & Mahon, J. 1956 \\
\hline & Porrocaecum ensicaudatum & Hawaii & van Riper, S. G., and C. van Riper. 1984 \\
\hline & Porrocaecum semiteres & Hawaii & van Riper, S. G., and C. van Riper. 1984 \\
\hline & Arythmorhynchus comptus & Alaska & Van Cleave, H. J., and R. Rausch. 1950 \\
\hline & Mediorhynchus orientalis & Hawaii & Schmidt, G. D., and R. E. Kuntz. 1977 \\
\hline & Plagiorhynchos charadrii & Hawaii & Amin, O. M., et al. 1999 \\
\hline \multicolumn{4}{|l|}{ Pacific Golden Plover NA } \\
\hline Pluvialis fulva & Echinostoma charadrii & USA & Doss, M. A., and M. M. Farr. 1969b \\
\hline \multicolumn{4}{|l|}{ Black-bellied Plover A } \\
\hline \multirow[t]{14}{*}{ Pluvialis squatarola (Squatarola) } & Cotylurus cornutus & Alaska & Bondarenko, S. K., and V. I. Kontrimavichus. 1999 \\
\hline & Echinoparyphium recurvatum & Bristol Bay, Alaska & Canaris, A. G., and J. M. Kinsella. 2000 \\
\hline & Echinoparyphium sp. & Florida & Forrester, D. J., and M. G. Spalding. 2003 \\
\hline & Mecynophallus glandulosus & Puerto Rico & Cable, R. M., et al. 1960 \\
\hline & Megalophallus reamesi & $\begin{array}{l}\text { Florida, British West Indies } \\
\text { Gulf of Mexico }\end{array}$ & Overstreet, R. M., and R. W. Heard III. 1995 \\
\hline & Microphallus claviformes & Puerto Rico & Cable, R. M., et al. 1960 \\
\hline & Microphallus sabanensis & Venezuela & Fernandes, B. M. M., et al. 2015 \\
\hline & Odhneria odhneri & Georgia & Sinclair, N. R. 1971 \\
\hline & Paramaritremopsis solielangi & Belize & Canaris, A. G., and J. M. Kinsella. 2001 \\
\hline & Parorchis holotestis & Puerto Rico & Cable, R. M., et al. 1960 \\
\hline & Probolocoryphe glandulosa & Puerto Rico & Yamaguti, S. 1971 \\
\hline & Psilochasmus sp. & Florida & Loftin, H. 1960 \\
\hline & Stephanoprora sp. & Florida & Loftin, H. 1960 \\
\hline & Stomylotrema fastosum & Brazil & Fernandes, B. M. M., et al. 2015 \\
\hline
\end{tabular}




\section{Table I. (cont.) Helminth parasites reported for the shorebird family Charadriidae from the Americas}

\begin{tabular}{|c|c|c|c|}
\hline Native hosts in the Americas & Parasite & Geographic location & Citation \\
\hline & Anomotaenia ericetorum & Bristol Bay, Alaska & Canaris, A. G., and J. M. Kinsella. 2000 \\
\hline & Aploparaksis diagonalis & Bristol Bay, Alaska & Canaris, A. G., and J. M. Kinsella. 2000 \\
\hline & Hymenolepis clandestina & Guadeloupe & Graber, M., and J. Euzeby. 1976b \\
\hline & Kowalewskiella cingulifera & Guadeloupe & Graber, M., and J. Euzeby. 1976b \\
\hline & Liga brevis & Bristol Bay, Alaska & Canaris, A. G., and J. M. Kinsella. 2000 \\
\hline & Liga sp. & Florida & Loftin, H. 1960 \\
\hline & Proterogynotaenia texanum & Texas; Belize & Huey, R. 1985; \\
\hline & & & Canaris, A. G., and J. M. Kinsella. 2001 \\
\hline & Proterogynotaenia variabilis & Bristol Bay, Alaska & Canaris, A. G., and J. M. Kinsella. 2000 \\
\hline & Schistocephalus solidus & Bristol Bay, Alaska & Canaris, A. G., and J. M. Kinsella. 2000 \\
\hline & Trichocephaloides beauporti & Guadeloupe & Graber, M., and J. Euzeby. 1976a \\
\hline & Wardium squatarolae & Alaska; Bristol Bay, Alaska & $\begin{array}{l}\text { Bondarenko, S. K., and V. I. Kontrimavichus. 1999; } \\
\text { Canaris, A. G., and J. M. Kinsella. } 2000\end{array}$ \\
\hline & Ancyracanthopsis winegardi & $\begin{array}{l}\text { Alberta; Manitoba; } \\
\text { Gulf of Mexico, USA }\end{array}$ & $\begin{array}{l}\text { Wong, P L., et al. 1990; } \\
\text { Anderson, R. C., et al. } 1996\end{array}$ \\
\hline & Chevreuxia americana & $\begin{array}{l}\text { Alberta, Manitoba; } \\
\text { California, Texas, USA, } \\
\text { Peru }\end{array}$ & Wong, P. L., and R. C. Anderson. 1991 \\
\hline & Desmidocerecella numidica & $\begin{array}{l}\text { British Columbia, Quebec, } \\
\text { New Brunswick }\end{array}$ & Wong, P. L., et al. 1990 \\
\hline & Eucoleus trilobus & Alaska & Bondarenko, S. K., and V. I. Kontrimavichus. 1999 \\
\hline & Sciadiocara bihamata & Alberta, Manitoba & Wong, P. L., et al. 1990 \\
\hline & Sciadiocara umbellifera & Alberta, Quebec; & Wong, P. L., et al. 1990; \\
\hline & & Washington, Texas, USA & Wong, P. L., and R. C. Anderson. 1991 \\
\hline & Skrjabinocerca prima & Alberta & Wong, P. L., et al. 1990 \\
\hline & Skrjabinoclava bartlettae & Alberta, Louisiana & Wong, P. L., and R. C. Anderson. 1990b \\
\hline & Skrjabinoclava tupacincai & Chile; Texas, Louisiana & $\begin{array}{l}\text { Wong, P. L., and R. C. Anderson. 1987; } \\
\text { Wong, P. L., et al. } 1990\end{array}$ \\
\hline & Skrjabinoclava wilsonae & Alberta & Wong, P. L., and R. C. Anderson. 1990b \\
\hline & Streptocara sp. & Alberta & Wong, P. L., et al. 1990 \\
\hline & Viktorocara capillaris & Alberta, Quebec, Texas; & Wong, P. L., et al. 1990; \\
\hline & & $\begin{array}{l}\text { Washington, California, } \\
\text { Louisiana }\end{array}$ & Wong, P. L., and R. C. Anderson. 1991 \\
\hline & Viktorocara limosae & Alberta, Manitoba, & Wong, P. L., et al. 1990; \\
\hline & & Quebec; California, USA & Wong, P. L., and R. C. Anderson. 1991 \\
\hline & Viktorocara schejkini & Canada & Gibson, G. G. 1972 \\
\hline & Polymorphus magnus & Bristol Bay, Alaska & Canaris, A. G., and J. M. Kinsella. 2000 \\
\hline
\end{tabular}

Pied Plover SA

Vanellus cayanus

(Hoploxypterus)

Southern Lapwing $M, C A, S A$

Vanellus chilensis
No reference found

Athesmia heterolecithodes Athesmia sp.

Echinostoma aphylactum

Leucochloridium parcum

Levinseniella cruzi

Neivaia cymbium

Stomylotrema tagax

Stomylotrema vicarium

Gyrocoelia perversa

Infula macrophallus

Infula sp.

Argentina
Brazil
Brazil
Brazil
Argentina; Mexico
Brazil
Brazil
Brazil; Argentina
Brazil
Brazil
Chile

Lunaschi, L. I., et al. 2007

Silveira, T. S., and C. Calegaro-Marques. 2016

Avancini, L. F. 2009

Silveira, T. S., and C. Calegaro-Marques. 2016

Martorelli, S. R., and V. A. Ivanov. 1996;

López, N. S. 1997

Avancini, L. F. 2009

Fernandes, B. M. M., et al. 2015

Avancini, L. F. 2009;

Fernandes, B. M. M., et al. 2015

Avancini, L. F. 2009

Silveira, T. S., and C. Calegaro-Marques. 2016

González-Acuña, D., et al. 2008 


\section{Table I. (cont.) Helminth parasites reported for the shorebird family Charadriidae from the Americas}

\begin{tabular}{|c|c|c|c|}
\hline Native hosts in the Americas & Parasite & Geographic location & Citation \\
\hline & Megalacanthus macracantha & Brazil & Bona, F. V. 1994 \\
\hline & Ancyracanthopsis coronata & Brazil & Vicente, J. J., et al. 1995 \\
\hline & Capillariidae & Brazil & Silveira, T. S., and C. Calegaro-Marques. 2016 \\
\hline & Dispharynx nasuta & Chile; Brazil & $\begin{array}{l}\text { González-Acuña, D., et al. 2008; } \\
\text { Silveira, T. S., and C. Calegaro-Marques. } 2016\end{array}$ \\
\hline & Heterakis psophiae & Brazil & Silveira, T. S., and C. Calegaro-Marques. 2016 \\
\hline & Synhimantus nasuta & Brazil & dos Santos, L. L., et al. 2020 \\
\hline & Centrorhynchus sp. & Brazil & Silveira, T. S., and C. Calegaro-Marques. 2016 \\
\hline & Gigantorhychida & Brazil & Silveira, T. S., and C. Calegaro-Marques. 2016 \\
\hline & Mediorhynchus sp. & Brazil & Silveira, T. S., and C. Calegaro-Marques. 2016 \\
\hline & Plagiorhynchus sp. & Chile; Brazil & González-Acuña, D., et al. 2008; \\
\hline & & & Silveira, T. S., and C. Calegaro-Marques. 2016 \\
\hline \multicolumn{4}{|l|}{ Andean lapwing $S A$} \\
\hline Vanellus resplendens & Aprocta ptiloscelidis & Argentina & Drago, F. B., et al. 2020 \\
\hline Vanellus sp. & Oxyspirura matogrosensis & Brazil & Tavares, L. E. R., et al. 2017 \\
\hline
\end{tabular}

\section{Vagrant and rare Charadriidae in the Americas}

Little Ringed Plover

Charadrius dubius

\section{Greater Sand-Plover}

Charadrius leschenaultii

Lesser Sand-Plover

Charadrius mongolus

Eurasian Dotterel

Charadrius morinellus

(Eudromias)

\section{Oriental Plover}

Charadrius veredus

Eurasian Golden Plover

Pluvialis apricaria

Northern Lapwing

Vanellus vanellus
No reference found

No reference found

No reference found

No reference found

No reference found

No reference found

No reference found
Vagrant to Caribbean Chesser, R. T., et al. 2019

and Alaska

Vagrant California, Florida Chesser, R. T., et al. 2019

Rare in Alaska

Paulson, D. 2005

Rare in Alaska, Paulson, D. 2005 vagrant Pacific Coast to Baja, California

Accidental/casual Chesser, R. T., et al. 2019

Exceptional vagrant to Chesser, R. T., et al. 2019 North America

Vagrant E. North America, Chesser, R. T., et al. 2019

Newfoundland, rare Alaska,

W to Ohio and S to Florida

and Georgia, Bermuda,

and the Caribbean 


\section{Table II. Trematode parasite-host list for shorebird family Charadriidae of the Americas}

Parasite

Acanthoparyphium pagollae

Athesmia heterolecithodes

Athesmia sp.

Collyriclum sp.

Cotylurus cornutus

Cyclocoelum lanceolatum

Cyclocoelum obscurum

Cyclocoelum triangularis

Echinochasmus sp.

Echinoparyphium aconiatum

Echinoparyphium baculus

Echinoparyphium recurvatum

Echinoparyphium sp.

Echinostoma aphylactum

Echinostoma charadrii

Echinostoma sp.

Gynaecotyla adunca

Gynaecotyla sippiwissettensis

Leucochloridium parcum

Leucochloridium propinqua

Levinseniella carcinidis

Levinseniella carteretensis

Levinseniella cruzi

Levinseniella hunteri

Levinseniella leptophallus

Levinseniella propinqua

Maritrema gratiosum

Maritrema ovatum

Maritreminoides patulum

Mecynophallus glandulosus

Megalophallus reamesi

Microphallus claviformes

Microphallus kinsellai

Microphallus quechuasensis

Microphallus sabanensis

Neivaia cymbium

Notocotylus attenuatus

Notocotylus chionis

Notocotylus stagnicolae

Odhneria odhneri

Paramaritremopsis solielangi

Paramonostomum actidis

Parorchis holotestis

Plagiorchis vitellatus

Probolocoryphe glandulosa

Pseudospelotrema charadrii

Psilochasmus sp.
Host

Charadrius wilsonia

Vanellus chilensis

Vanellus chilensis

Charadrius vociferous

Pluvialis squatarola

Charadrius vociferous

Charadrius semipalmatus, C. vociferous

Charadrius vociferous

Charadrius semipalmatus

Charadrius vociferous

Charadrius vociferous

Pluvialis squatarola

Pluvialis squatarola

Vanellus chilensis

Pluvialis fulva

Charadrius vociferous

Charadrius wilsonia

Charadrius melodus

Vanellus chilensis

Charadrius sp.

Charadrius semipalmatus

Charadrius wilsonia

Charadrius semipalmatus, Vanellus chilensis

Charadrius wilsonia

Charadrius wilsonia

Pluvialis dominica

Charadrius semipalmatus

Charadrius semipalmatus

Charadrius wilsonia

Charadrius wilsonia, Pluvialis squatarola

Charadrius wilsonia, Pluvialis squatarola

Charadrius semipalmatus, C. wilsonia, Pluvialis squatarola

Charadrius semipalmatus

Charadrius vociferous

Pluvialis squatarola

Charadrius wilsonia, Vanellus chilensis

Charadrius vociferous

Charadrius falklandicus

Charadrius semipalmatus

Charadrius falklandicus, C. wilsonia, Pluvialis squatarola

Charadrius nivosus (and alexandrinus), C. semipalmatus, Pluvialis squatarola

Charadrius hiaticula, C. semipalmatus, C. wilsonia

Charadrius wilsonia, Pluvialis squatarola

Charadrius vociferous

Pluvialis squatarola

Charadrius wilsonia

Pluvialis squatarola 
Table II. Trematode parasite-host list for shorebird family Charadriidae of the Americas

\begin{tabular}{ll} 
Parasite & Host \\
\hline Spelotrema excellens & Charadrius sp. \\
Stephanoprora sp. & Pluvialis squatarola \\
Stomylotrema fastosum & Pluvialis squatarola \\
Stomylotrema tagax & Vanellus chilensis \\
Stomylotrema vicarium & Charadrius vociferous, Vanellus chilensis \\
Tanaisia fedtschenkoi & Charadrius sp., C. vociferous \\
Tanaisia valida & Charadrius wilsonia \\
Transcoelom sp. & Charadrius vociferous \\
Uvitellina macroisophaga & Charadrius vociferous \\
Uvitellina psuedocotylea & Charadrius vociferous \\
Zygocotyle lunata & Charadrius wilsonia
\end{tabular}


Table III. Cestode parasite-host list for shorebird family Charadriidae from the Americas

\begin{tabular}{|c|c|}
\hline Parasite & Host \\
\hline Anomotaenia bacilligera & Charadrius vociferous \\
\hline Anomotaenia ericetorum & Pluvialis squatarola \\
\hline Anomotaenia filovata & Charadrius vociferous \\
\hline Aploparaksis brachyphallos & Charadrius hiaticula \\
\hline Aploparaksis diagonalis & Pluvialis squatarola \\
\hline Aploparaksis sp. & Charadrius vociferous \\
\hline Choanotaenia macracantha & Charadrius vociferous \\
\hline Choanotaenia sp. & Charadrius vociferous \\
\hline Gyrocoelia crassa & Charadrius collaris \\
\hline Gyrocoelia fuhrmani & Charadrius collaris \\
\hline Gyrocoelia miligani & Charadrius nivosus (and alexandrinus), C. vociferous \\
\hline Gyrocoelia pagollae & Charadrius wilsonia \\
\hline Gyrocoelia perversa & Vanellus chilensis \\
\hline Hymenolepis clandestina & Pluvialis squatarola \\
\hline Hymenolepis collariella & Charadrius collaris \\
\hline Hymenolepis hughesi & Charadrius collaris, C. melodus, C. semipalmatus \\
\hline Infula macrophallus & Vanellus chilensis \\
\hline Infula sp. & Vanellus chilensis \\
\hline Kowalewskiella cingulifera & Pluvialis squatarola \\
\hline Liga brevis & Pluvialis squatarola \\
\hline Liga sp. & Pluvialis squatarola \\
\hline Megalacanthus macracantha & Vanellus chilensis \\
\hline Megalacanthus sp. & Charadrius vociferous \\
\hline Monopylidium sp. & Charadrius vociferous \\
\hline Nadejdolepis litoralis & Charadrius nivosus (and alexandrinus) \\
\hline Nadejdolepis paranitidulans & Charadrius nivosus (and alexandrinus) \\
\hline Paraliga charadrii & Charadrius semipalmatus \\
\hline Paraprogynotaenia jimenezi & Charadrius wilsonia \\
\hline Progynotaenia americana & Charadrius melodus, C. semipalmatus, C. vociferous, Pluvialis dominica \\
\hline Proterogynotaenia neoarctica & Charadrius vociferous \\
\hline Proterogynotaenia odhneri & Charadrius vociferous \\
\hline Proterogynotaenia texanum & Pluvialis squatarola \\
\hline Proterogynotaenia variabilis & Pluvialis squatarola \\
\hline Schistocephalus solidus & Pluvialis squatarola \\
\hline Shipleyia inermis & Charadrius nivosus (and alexandrinus) \\
\hline Taenia charadrii & Charadrius collaris \\
\hline Trichocephaloides beauporti & Pluvialis squatarola \\
\hline Wardium squatarolae & Pluvialis squatarola \\
\hline
\end{tabular}




\section{Table IV. Nematode parasite-host list for shorebird family Charadriidae of the Americas}

\begin{tabular}{|c|c|}
\hline Parasite & Host \\
\hline Ancyracanthopsis coronata & Vanellus chilensis \\
\hline Ancyracanthopsis winegardi & Charadrius wilsonia, Pluvialis squatarola \\
\hline Aprocta ptiloscelidis & Vanellus resplendens \\
\hline Capillaria contorta & Charadrius vociferous, Charadrius sp. \\
\hline Capillariidae & Vanellus chilensis \\
\hline Chevreuxia americana & Charadrius melodus, C. semipalmatus, C. vociferous, Pluvialis squatarola \\
\hline Chevreuxia sp. & Charadrius vociferous \\
\hline Chevreuxia vanelli & Charadrius vociferous \\
\hline Desmidocerecella numidica & Pluvialis squatarola \\
\hline Dispharynx nasuta & Charadrius vociferous, Vanellus chilensis \\
\hline Dispharynx spiralis & Charadrius vociferous \\
\hline Eucoleus trilobus & Pluvialis squatarola \\
\hline Eulimdana lari & Charadrius hiaticula \\
\hline Eulimdana sonni & Charadrius hiaticula \\
\hline Heterakis psophiae & Vanellus chilensis \\
\hline Oxynema sp. & Oreopholus ruficollis \\
\hline Oxyspirura matogrosensis & Vanellus sp. \\
\hline Oxyspirura sp. & Charadrius vociferous \\
\hline Porrocaecum ensicaudatum & Charadrius sp., Pluvialis dominica \\
\hline Porrocaecum falklandicus & Charadrius modestus \\
\hline Porrocaecum semiteres & Pluvialis dominica \\
\hline Schistorophus laciniatus & Charadrius vociferous \\
\hline Sciadiocara bihamata & Pluvialis squatarola \\
\hline Sciadiocara umbellifera & Charadrius collaris, C. wilsonia, Pluvialis squatarola \\
\hline Skrjabinocerca prima & Charadrius vociferous, Pluvialis squatarola \\
\hline Skrjabinoclava bartlettae & Pluvialis squatarola \\
\hline Skrjabinoclava horrida & Charadrius semipalmatus, C. wilsonia \\
\hline Skrjabinoclava morrisoni & Charadrius semipalmatus \\
\hline Skrjabinoclava pusillae & Charadrius semipalmatus, C. wilsonia \\
\hline Skrjabinoclava semipalmatae & Charadrius semipalmatus, C. wilsonia \\
\hline Skrjabinoclava sp. & Charadrius semipalmatus \\
\hline Skrjabinoclava tupacincai & Charadrius semipalmatus, Pluvialis squatarola \\
\hline Skrjabinoclava wilsonae & Charadrius wilsonia, Pluvialis squatarola \\
\hline Spirurid larvae & Charadrius vociferous \\
\hline Spiruroidean nematode & Charadrius vociferous \\
\hline Stellocaronema skrjabini & Charadrius collaris, C. semipalmatus, C. vociferous \\
\hline Streptocara sp. & Charadrius vociferous, Pluvialis squatarola \\
\hline Strongyloides sp. & Charadrius vociferous \\
\hline Synhimantus nasuta & Vanellus chilensis \\
\hline Tetrameres megaphasmidiata & Charadrius falklandicus \\
\hline Tropisurus nouveli & Charadrius wilsonia \\
\hline Viktorocara capillaris & Charadrius semipalmatus, C. vociferous, C. wilsonia, Pluvialis squatarola \\
\hline Viktorocara limosae & Charadrius semipalmatus, Pluvialis squatarola \\
\hline Viktorocara schejkini & Charadrius wilsonia, Pluvialis squatarola \\
\hline
\end{tabular}


Table V. Acanthocephala parasite-host list for shorebird family Charadriidae of the Americas

\begin{tabular}{ll} 
Parasite & Host \\
\hline Acanthocephalan larvae & Charadrius vociferous \\
Arythmorhynchus comptus & Pluvialis dominica \\
Centrorhynchus sp. & Vanellus chilensis \\
Gigantorhychida & Vanellus chilensis \\
Mediorhynchus orientalis & Pluvialis dominica \\
Mediorhynchus papillosus & Charadrius vociferous \\
Mediorhynchus robustus & Charadrius vociferous \\
Mediorhynchus sp. & Vanellus chilensis \\
Plagiorhynchus charadrii & Charadrius semipalmatus, Pluvialis dominica \\
Plagiorhynchus crassicolle & Charadrius wilsonia \\
Plagiorhynchus sp. & Vanellus chilensis \\
Polymorphus magnus & Pluvialis squatarola \\
Polymorphus minutus & Charadrius vociferous
\end{tabular}


No. 15. Canaris et al., Helminth Parasites from Shorebirds from the Americas

Tables VI. Summary data compiled from Tables I-V and Literature Cited

\section{A. Hosts and number of species}

\begin{tabular}{lcccccc} 
Host $(n=21)$ & Trematode & Cestode & Nematode & Acanthocephala & Total & $\%$ \\
\hline Charadrius vociferous & 15 & 11 & 15 & 4 & 45 & 23 \\
Pluvialis squatarola & 14 & 11 & 14 & 1 & 40 & 20 \\
Charadrius wilsonia & 16 & 2 & 9 & 1 & 28 & 14 \\
Charadrius semipalmatus & 11 & 3 & 10 & 1 & 25 & 13 \\
Vanellus chilensis & 8 & 4 & 5 & 4 & 21 & 11 \\
Charadrius collaris & 0 & 5 & 2 & 0 & 7 & 4 \\
Pluvialis dominica & 1 & 1 & 2 & 3 & 7 & 4 \\
Charadrius nivosus (and alexandrinus) & 1 & 4 & 0 & 0 & 5 & 3 \\
Charadrius sp. & 3 & 0 & 2 & 0 & 5 & 3 \\
Charadrius hiaticula & 1 & 1 & 2 & 0 & 4 & 2 \\
Charadrius melodus & 1 & 2 & 1 & 0 & 4 & 2 \\
Charadrius falklandicus & 2 & 0 & 1 & 0 & 3 & 2 \\
Charadrius modestus & 0 & 0 & 1 & 0 & 1 & 1 \\
Oreopholus ruficollis & 0 & 0 & 1 & 0 & 1 & 1 \\
Pluvialis fulva & 1 & 0 & 0 & 0 & 1 & 1 \\
Vanellus resplendens & 0 & 0 & 1 & 0 & 1 & 1 \\
Vanellus sp. & 0 & 0 & 1 & 0 & 1 & 1 \\
Charadrius alticola & 0 & 0 & 0 & 0 & 0 & 0 \\
Charadrius montanus & 0 & 0 & 0 & 0 & 0 & 0 \\
Phegornis mitchellii & 0 & 0 & 0 & 0 & 0 & 0 \\
Vanellus cayanus & 0 & 0 & 0 & 0 & 0 & 0 \\
& 74 & 44 & 67 & 14 & 199 & $100 \%$ \\
\hline
\end{tabular}

VI. B. Number and percentage of helminth species for the four taxa

Number of helminth species

Percent

\begin{tabular}{lcc}
\hline Trematodes & 58 & 38 \\
Cestodes & 38 & 25 \\
Nematodes & 44 & 29 \\
Acanthocephala & 13 & 9 \\
Total & 153 & $100 \%$ \\
\hline
\end{tabular}


Tables VI. (cont.) Summary data compiled from Tables I-V and Literature Cited

\section{C. Geographic locations of infected hosts}

\begin{tabular}{lc} 
Location & Number of infected hosts \\
\hline USA & 144 \\
Canada & 59 \\
South America & 44 \\
Puerto Rico & 17 \\
Alaska & 12 \\
Central America & 12 \\
Cuba & 10 \\
North America & 7 \\
Guadeloupe & 6 \\
Hawaii & 4 \\
Mexico & 3 \\
Antigua & 1 \\
West Indies & 1 \\
\hline
\end{tabular}

Note: American states and Canadian provinces are summed under USA and Canada, respectively.

\section{D. Host-parasite citations per decade}

\begin{tabular}{lc} 
Decade & Number of citations \\
\hline $1930-39$ & 1 \\
$1940-49$ & 2 \\
$1950-59$ & 9 \\
$1960-69$ & 14 \\
$1970-79$ & 12 \\
$1980-89$ & 7 \\
$1990-1999$ & 19 \\
$2000-2009$ & 8 \\
$2010-2020$ & 6 \\
\hline
\end{tabular}




\section{Literature Cited}

Amin, O. M., A. G. Canaris, and J. M. Kinsella. 1999. A taxonomic reconsideration of the genus Plagiorhynchus s. lat. (Acanthocephala: Plagiorhychidae), with descriptions of South African Plagiorhynchus (Prosthorhynchus) cylindraceus from shore birds and $P$. (P.) malayensis, and a key to the species of the subgenus Prosthorhynchus. Journal of Helminthology 66:123-132.

Anderson, R. C., P. L. Wong, and C. M. Bartlett. 1996. The Acuarioid and Habronematoid nematodes (Acuarioidea, Habronematoidea) of the upper digestive tract of waders: a review of observations on their host and geographic distributions and transmission in marine environments. Parasite 4:303-312.

Avancini, L. F. 2009. Identification of helminths and arthropods in Vanellus chilensis (Molina, 1782) (Charadriiformes: Charadriidae), lapwing in the south region of the Rio Grande do Sul state. MS thesis. Universidade Federal De Pelotas, Pelotas, Brazil. 58 pp.

Baer, J. G. 1940. Some avian tapeworms from Antigua. Parasitology 32:174-197.

Bartlett, C. M. 1992. New, known, and unidentified species of Eulimdana (Nematoda): additional information on biologically unusual filarioids of charadriiform birds. Systematic Parasitology 23:209-230.

Barus, V., and N. L. Hernandez. 1971. Nemátodos Parásitos de Aves en Cuba. Parte IV. serie Poeyana, Instituto de Biologia, Academia de Ciencias de Cuba 88:1-75.

Blaylock, R. B. 1989. Metazoan parasite community composition and structure of killdeer, Charadrius vociferous L., along the upper Rio Grande Valley. Unpublished thesis. University of Texas at El Paso, Texas. 105 pp.

Bona, F. V. 1994. Family Dilepididae Ralliet \& Henry, 1909. In: Keys to the cestode parasites of vertebrates. Ed. by L. F. Khalil, A. Jones, and R. A. Bray. CAB International.

Bondarenko, S. K., and V. I. Kontrimavichus. 1999. The helminth fauna of Charadriiformes in Alaska. Zoologicheskiĭ zhurnal 78:643-653.

Byrd, E. E., and J. F. Denton. 1950. The helminth parasites of birds. I. A review of the trematode genus Tanasia Skrjabin, 1924. American Midland Naturalist 43:32-57.

Caballero, C. E., and H. N. Ibáñez. 1970. Estudios helminthlogicos de la Republica del Peru. I. Dos especies de trematoda de la familia Microphallidae Travassos, 1920 que parasitan a Aves. Anales del Instituto de Biologia, Universidad Nacional Autónoma México Zoologia 41:29-38.

Cable, R. M., and M. E. Myers. 1956. A dioecious species of Gyrocoelia (Cestoda: Acoelidae) from the naped plover. Journal of Parasitology 42:510-515.
Cable, R. M., R. S. Connor, and J. E. Balling. 1960. Digenetic trematodes of Puerto Rico. Scientific Survey of Porto Rico and the Virgin Islands 17:187-255.

Canaris, A. G., and J. M. Kinsella. 2000. Helminth parasites in six species of shorebirds (Charadrii) from Bristol Bay, Alaska. Comparative Parasitology 67:250-252.

Canaris, A. G., and J. M. Kinsella. 2001. Helminth parasites of six species of shorebirds (Charadrii) from the coast of Belize. Memórias do Instituto Oswaldo Cruz 96:827-830.

Capasso, S., V. D'Amico, and J. I. Diaz. 2017. Odhneria odhneri Travassos, 1921 (Trematoda: Microphallidae) in migrant shorebirds from Patagonia, Argentina. Revista Argentina de Parasitology 6:15-20.

Chesser, R. T., Burns, K. J., Cicero, C., Dunn, J. L., Kratter, A. W., Lovette, I. J., Rasmussen, P. C., Remsen, J. V., Stotz, D. F., and Winker, K. 2019. Sixtieth supplement to the American Ornithological Society's check-list of North American Birds. The Auk. 20:1-23.

Ching, H. L. 1965. Systematic notes on some North American microphallid trematodes. Proceedings of the Helminthological Society of Washington. 32:138-140.

Clark, D. T. 1952. Three new dilepidid cestodes, Dictymetra numenii n. gen. n. sp., Dictymetra paranumenii n. sp., and Anomotaenia filovata n. sp. Proceedings of the Helminthological Society of Washington 19:18-27.

Coil, W. H. 1956. Two new hymenolepis cestodes from Mexican birds with observations on Hymenolepis crocethiae Webster, 1947. Journal of Parasitology 42:584587.

Coil, W. H. 1963. The genera Gyrocoelia Fuhrmann, 1899 and Infula Burt, 1939 with observations on the histochemistry of egg membranes. Proceedings of the Helminthological Society of Washington 30:111-117.

Coil, W. H., and R. Heard. 1966. Levinseniella carteretensis sp. nov., a microphallid trematode from the Wilson Plover, Charadrius wilsonia. Proceedings of the Helminthological Society of Washington 33:54-56.

Cremonte, F. M., C. Digiani, L. O. Bala, and G. T. Navone. 2001. Tetrameres (Tetrameres) megaphasmidiata n. sp. (Nematoda: Tetrameridae), a parasite of the two-banded plover, Charadrius falklandicus, and white-rumped sandpiper, Calidris fuscicollis from Patagonia, Argentina. Journal of Parasitology 87:148-151.

Deblock, S., and A. G. Canaris. 2000. Trois Nadejdolepis Spasskii \& Spasskaya, 1954 (Cestoda: Hymenolepididae) parasites de Charadrii (Aves) du Bélize. Systematic Parasitology 47:193-201.

Deblock, S., and F. Rosé. 1962. Les Hymenolepis (sensu lato) de Charadriiformes. Annals Parasitologie Humaine et Comparée 37:767-847.

del Valle, M. T., and A. Coy-Otero. 1990. New records of helminths in Cuban birds. Poeyana, La Habana 401:1-4. 
Didyk, A. S., and M. D. B. Burt. 1998. Paraliga charadrii n. sp. (Cestoda: Dilepididae) from the semipalmated plover, Charadrius semipalmatus Bonaparte (Aves: Charadriiformes). Journal of Parasitology 84:828-830.

dos Santos, L. L., C. Silveira Mascarenhas, P. R. Silveira-dos Santos, and N. A da Rosa-Farias. 2020. Helminth fauna of Passer domesticus (Passeriformes: Passeridae) in the southern extreme of Brazil. Revista Mexicana de Bioversidad 91:(2020):19 Mayo.

Doss, M. A., and M. M. Farr. 1969a.Trematoda and trematode diseases. Part 12: Hosts: genera A-L. Index-Catalogue of Veterinary Zoology. United States Government Printing Office, Washington. 377 pp.

Doss, M. A., and M. M. Farr. 1969b. Trematoda and trematode diseases. Part 12: Hosts: genera M-Z. Index-Catalogue of Veterinary Zoology. United States Government Printing Office, Washington. 694 pp.

Drago, F. B., V. Núñez, and M. D. Díaz. 2020. Checklist of the nematodes of wild birds of Argentina. Revue suisse de Zoologie 127:43-46.

Eckman, M. K. 1968. Helminth parasites of the killdeer in Colorado. Journal of Parasitology 54:1143.

Fernandes, B. M. M. 1976. Sobre as especies brasileiras da familia Cyclocoelidae Kossack 1911 (Trematoda, Cyclocoelidae). Memórias do Instituto Oswaldo Cruz 74:289-294.

Fernandes, B. M. M., J. Marcia, M. Q. Cardenas, and S. Cohen. 2015. South American trematodes parasites of birds and mammals. Edition: 1 Oficina do Livro. 550 pp.

Forrester, D. J., and M. G. Spalding. 2003. Parasites and Diseases of Wild Birds in Florida. University Press of Florida, Gainesville. 1152 pp.

Gibson, G. G. 1972. Sciadiocara denticulata n. sp. (Acuariidae) from Actitis macularia (L.) and other nematodes from spotted sandpiper and black bellied plover. Canadian Journal of Zoology 50:131-136.

González-Acuña, D., P. Olmedo, and A. Cicchino. 2008. Parásitos de Vanellus chilensis (Aves, Charadriidae) en Chillán, centrosur de Chile. Boletín Chileno de Chile 14:36-48.

Graber, M., and J. Euzeby. 1976a. Trichocephaloides beauporti n. sp. Cestodes nouveau des Charadriiformes et de certains Passériformes de la Guadeloupe. Annales de Parasitology (Paris) 51:189-198.

Graber, M., and J. Euzeby. 1976b. Deuxième enquête parasitologique en Guadeloupe. Note 2: Les cestodes des oiseaux aquatiques. Bulletin de la Societe des Sciences Veterinaires et de Medecine Comparee de Lyon 78:153-171.

Hannum, C. A., and B. E. Wilson. 1934. Uvitellina macroisophaga $\mathrm{n}$. sp. from the killdeer. Transactions of the American Microscopical Society 53:245-250.
Hartwich, G., and I. Kilias. 1992. Die typen der Cercomeromorphae (Platyhelminthes) des Zoologischen Museums in Berlin. Mitteilungen aus Zoologischen Museum in Berlin 68:209-248.

Hayman, P., J. Marchant, and T. Prater. 1986. Shorebirds: An identification guide to the waders of the world. Houghton Mifflin Company, Boston. 412 pp.

Heard III, R. W. 1968. Levinseniella hunteri sp. nov. anew species of microphallid trematode from the Wilson's plover, Charadrius wilsonia Ord. Proceedings of the Helminthological Society of Washington 35:41-43.

Hoberg, E. P. 1986. Eulimdana rauschorium n. sp., a filarioid nematode (Lemdaninae) fromLarus dominicanus in Antarctica, with comments on Evolution and Biogeography. Journal of Parasitology 72:755-761.

Huey, R. 1985. Proterogynotaenia texanum sp. n. (Cestoidea: Progynotaeniidae) from the Black-bellied Plover, Pluvialis squatarola. Proceedings of the Helminthological Society of Washington 52:41-43.

Jensen, D. N. 1971. Eucotyle castenia n. sp. and other members of the family Eucotylidae from birds in British Columbia. Canadian Journal of Zoology 49:1053-1058.

Loftin, H. 1960. An annotated check-list of trematodes and cestodes and their vertebrate hosts from Northwest Florida. Florida Academy of Sciences Quarterly Journal 23:302-314.

López, N. S. 1997. Descripción de tremátodos digenéticos del pez Bascanichtys peninsulae (Gilbert, 1891) de las Islas Marías, México. MS thesis, Universidad Autonoma De Nuevo Leon, San Nicolas De Los Garza, Nuevo Leon. $55 \mathrm{pp}$.

Lunaschi, L. I., F. Cremonte, and F. B. Drago. 2007. Checklist of digenean parasites of birds from Argentina. Zootaxa 1403:1-36.

Macko, J. K. 1969. Further knowledge on the variability of Cyclocoelum obscurum (Leidy, 1887) Trematoda. Biologia 24:606-616.

Macko, J. K., and M. Spakulova. 1998. Taxonomic study of Progynotaenia odhneri isolated from Charadrius vociferous ternominatus from Cuba. Acta Parasitologica 43:59-63.

Mahon, J. 1956. On a collection of avian cestodes from Canada. Canadian Journal of Zoology 34:104-109.

Martorelli, S. R., and V. A. Ivanov. 1996. Host-induced and geographical variation in Levinseniella cruzi Travassos, 1920 (Digenea: Microphallidae). Journal of the Helminthological Society of Washington 63:130-135.

Overstreet, R. M., and R. W. Heard III. 1995. A new species of Megalophallus (Digenea: Microphallidae) from the clapper rail, other birds, and a littoral isopod Ligia baudiniana. Canadian Journal of Fisheries and Aquatic Sciences 52:98-104. 
Paulson, D. 2005. Shorebirds of North America. Princeton University Press, Princeton, NJ. 361 pp.

Rêgo, A. A. 1968. Sôbre três cestódeos de aves Charadriiformes. Memórias do Instituto Oswaldo Cruz 66:107-115.

Rietschel, G., and B. Werding. 1978. Trematodes of birds from northern Colombia. Zeitschrift für Parasitenkunde $57: 57-82$

Riggins, M. A. 1953. A study of the helminth parasites of certain shore inhabiting birds from Lake Texoma, Oklahoma. Proceedings of the Oklahoma Academy of Science 34:57-59.

Rysavy, B. 1996. Nuevas especies de Céstodos (Cestoda: Cyclophyllidea) de aves para Cuba. Poeyana, Serie A 19:1-22.

Schmidt, G. D., and R. E. Kuntz. 1977. Revision of Mediorhynchus Van Cleave 1916 (Acanthocephala) with a key to species. The Journal of Parasitology 63:500-507.

Silveira, T. S., and C. Calegaro-Marques. 2016. Helminth parasite diversity discloses age and sex differences in the foraging behavior of southern lapwings (Vanellus chilensis). Austral Ecology 41:465-591.

Sinclair, N. R. 1971. A reviewal of Odhneria odhneri Travassos, 1921 (Trematoda: Microphallidae). Journal of Parasitology 57:980-982.

Tavares, L. E. R., K. M. Campiāo, R. Costa-Pereira, and F. Paiva. 2017. Helminth endoparasites of wildlife vertebrates in Mato Grosso do Sul, Brazil. Iheringia Série Zoologia 107: (suppl.)

Thatcher, V. E. 1993. Trematódeos neotropicais. Instituto Nacional de Pesquisas da Amazonia. Presidencia de la República. Ministerio de Ciencia e Technologia. Calderaro. 533 pp.

Van Cleave, H. J., and R. Rausch. 1950. A new species of the Acanthocephalan genus Arythmorhynchus from sandpipers of Alaska. Journal of Parasitology 36:278-283.

van Riper, S. G., and C. van Riper. 1984. A summary of known parasites and diseases recorded from the Avifauna of the Hawaiian Islands. Pages 298-357. In: Hawaii's terrestrial ecosystems preservation and management. Ed. by C. P. Stone and J. M. Scott. Cooperative National Park Resources Studies Unit, University of Hawaii, Honolulu.

Vicente, J. J., H. O. Rodrigues, D. C. Gomes, and R. M. Pinto. 1995. Nematóides do Brasil. Parte IV: nematóides de aves. Revista Brasileira de Zoologia 12:273.

Weatherly, N. F., and A. G Canaris. 1961. Some parasites of Washington and Oregon vertebrates. Journal of Parasitology 47:230.

Webster, J. D. 1947. Studies on the genus Hymenolepis, with descriptions of three new species. Journal of Parasitology 33:99-106.
Webster, J. D. 1951. Systematic notes on the North American Acoelidae (Cestoda). Journal of Parasitology 37:111-118.

Whittaker, F. H., G. D. Schmidt, and J. G. Diaz. 1970. Helminth parasites of some birds in Puerto Rico. Proceedings of the Helminthological Society of Washington 37:123-124.

Winkler, D. W., S. M. Billerman, and I. J. Lovette. 2020. Plovers and Lapwings (Charadriidae), version 1.0. In: Birds of the World. Ed. by S. M. Billerman, B. K. Keeney, and T. S. Schulenberg. Cornell Lab of Ornithology, Ithaca, NY, USA. https://doi/10.2173/bow.charad1.01

Wong, P. L., and R. C. Anderson. 1983. Revision of the genera Cordonema Schmidt and Kuntz, 1972 and Skrjabinoclava Sobolev, 1943 (Nematoda: Acuarioidea). Canadian Journal of Zoology 61:339-348.

Wong, P. L., and R. C. Anderson. 1987. New and described species of Skrjabinoclava Sobolev, 1943 (Nematoda: Acuarioidea) of the proventriculus of nearctic waders (Aves: Charadriiformes) with a review of the genus and key to the species. Canadian Journal of Zoology 65:27602779.

Wong, P. L., and R. C. Anderson. 1988. Skrjabinoclava bartlettae n. sp. (Nematoda: Acuarioidea) from the blackbellied plover (Pluvialis squatarola) (L.) (Charadriiformes: Charadriidae). Canadian Journal of Zoology 66:2202-2264.

Wong, P. L., and R. C. Anderson. 1990a. Ancyracanthopsis winegardi n. sp. (Nematoda: Acuarioidea) from Pluvialis squatarola (Aves: Charadriidae) and Acyracanthus heardi n. sp. from Rallus longirostris (Aves: Rallidae), and a review of the genus. Canadian Journal of Zoology 68:1297-1306.

Wong, P. L., and R. C. Anderson. 1990b. Host and geographic distribution of Skrjabinoclava spp. (Nematoda: Acuarioidea) in Nearctic shorebirds (Aves: Charadriiformes), and evidence for transmission in marine habitats in staging and wintering areas. Canadian Journal of Zoology 68:2539-2552.

Wong, P. L., and R. C. Anderson. 1991. Distribution of gizzard nematodes (Habronematoidea, Acuarioidea) of new world shorebirds (Charadriiformes), with special reference to localities of transmission. Canadian Journal of Zoology 69:2579-2588.

Wong, P. L., C. M. Bartlett, L. N. Measures, M. A. McNeill, and R. C. Anderson. 1990. Nematodes of Birds: Synopsis of the Parasites of Vertebrates of Canada. Queen's Printer, Edmonton, $A B$, Canada. 44 pp.

Yamaguti, S. 1959. Systema helminthum. Vol. II. The cestodes of vertebrates. Interscience Publishers, New York. 860 pp.

Yamaguti, S. 1961. Systema helminthum. Vol. III. The nematodes of vertebrates. Part 1 Interscience Publishers, New York. 679 pp.

Yamaguti, S. 1971. Synopsis of Digenetic Trematodes of Vertebrates. Vol. 1. Keigaku Publishing Company, Tokyo. 1074 pp. 